Proceedings

\title{
Probiotic Lactobacillus Reuteri Growth Improved under Fucoidan Exposure ${ }^{+}$
}

\author{
Neus Ricós-Muñoz, Sergi Maicas and María Consuelo Pina-Pérez *
}

Citation: Ricós-Muñoz, N.

Maicas, S.; Pina-Pérez, M.C.

Probiotic Lactobacillus Reuteri

Growth Improved under Fucoidan Exposure. Proceedings 2021, 70, 106. https://doi.org/10.3390/foods_202007724

Published: 10 November 2020

Publisher's Note: MDPI stays neutral with regard to jurisdictional claims in published maps and institutional affiliations.

Copyright: $@ 2020$ by the authors. Licensee MDPI, Basel, Switzerland. This article is an open access article distributed under the terms and conditions of the Creative Commons Attribution (CC BY) license (http://creativecommons.org/licenses/by/4.0/).

\author{
Departament de Microbiologia i Ecologia, Universitat de València, 46100 Burjassot, Spain; \\ neusrimu@alumni.uv.es (N.R.-M.); sergi.maicas@uv.es (S.M.) \\ * Correspondence: maria.c.pina@uv.es; Tel.: +34-96-354-4059 \\ + Presented at the 1st International Electronic Conference on Food Science and Functional Foods, \\ 10-25 November 2020; Available online: https://foods_2020.sciforum.net/.
}

\begin{abstract}
Allergy is the most common chronic disease in Europe and about $40 \%$ of the population suffers from it. Microbiota are closely linked to the immune system, with some probiotics characterized as beneficial, contributing to reductions in allergenic symptomatology. To ensure the predominance of beneficial probiotics in the gut microbiota, the use of prebiotics is very important. Among the most important prebiotics are those of marine origin, particularly the fucoidan, a polysaccharide from brown algae (Phaeophyta). The effect of fucoidan on Lactobacillus reuteri in vitro growth behavior has been studied using turbidimetry, plate count and statistical analysis. The fucoidan revealed a significant prebiotic effect, increasing L. reuteri growth $(\approx 2 \log 10$ cycles after $48 \mathrm{~h}$ exposure). The combination of the prebiotic effect of the fucoidan with the direct action that it exerts on allergy and other health problems make this molecule a promising nutraceutical for future use.
\end{abstract}

Keywords: fucoidan; prebiotic; Lactobacillus reuteri; allergy

\section{Introduction}

Allergy is the most common chronic disease in Europe and about $40 \%$ of the population suffers from it. Microbiota play a key role in the induction, control and function of the immune system. There is a symbiotic relationship between microbiota and the immune system, so the immune system evolves as the complexity of microbiota increases [1]. The gut microbiota (GM), although this is specific to each human being, consist mainly of four large phyla: Firmicutes (gram-positive), Bacteroides (gram-negative), Actinobacteria (gram-positive) and Proteobacteria (gram-negative) [2,3]. An imbalance (dysbiosis) in the percentages of each phylum translates into several changes and manifestations that alter the metabolic function of the immune system, which ends up generating different diseases, such as food allergies [3,4].

The diversity of GM in an individual changes from her/his birth to her/his maturity. The changes are influenced by genetics, epigenetics, and environmental factors. The GM differs, during the first months of life, in terms of both composition and diversity in children with or without allergic diseases. Children allergic to nuts have a microbiota with increased Bacteroides spp. and children allergic to cow's milk show a decrease in the phylum Firmicutes [4]. In experimentation animals, it is observed that an alteration of the microbiota affecting the families Porphyromonadaceae, Lactobacillaceae, Rikenellaceae and Lachnospiraceae may be behind the allergy to ovalbumin [5]. Li et al. [6] demonstrated that Lactobacillus reuteri is the most effective probiotic strain to reduce respiratory track inflammation, since it reduces the total $\operatorname{IgE}$ and the production of Th2-associated inflammatory cytokines. 
Prebiotics are typically polymers of carbohydrates that cannot be digested or absorbed by the human gut, although there are other compounds, such as alcohols, enzymes, peptides, unsaturated fatty acids, amino acids, or organic acids [7]. Algae are important sources of polysaccharides (PS) and oligosaccharides (OS), which have attracted great interest in terms of their prebiotic potential (alginates, carrageenans, exopolysaccharides, fucans and fucoids). Enzymes in the upper gastrointestinal tract do not break down PS from seaweed, so this PS can be used for food prebiotics and to enhance the growth of lactic acid bacteria such as Lactobacillus spp. and Bifidobacterium spp. [7,8]. Moreover, the PS from algae can be digested and fermented by GM to produce Short Chain Fatty Acids (SCFAs) which can improve the epithelial barrier function and cardiovascular health [9].

A very interesting prebiotic is fucoidan, which includes a group of PS from the cell wall of some brown seaweeds and some invertebrate marine animals. The fucoidan from the seaweed Fucus vesiculosus contains L-fucose and sulfate [10] and can act as an anticoagulant and anti-thrombotic, antimicrobial, antitumor and immunomodulatory, antioxidant, blood lipid reducer, anti-inflammatory, gastric protector, hepatoprotector and prebiotic $[10,11]$. As a result of its structure, fucoidan cannot be digested by gastric and pancreatic enzymes, so it reaches the small bowel unchanged, where it can have a favourable effect on GM [12]. Fucoidan also promotes an enrichment in Lactobacillus spp. of pig colon [13] and stimulates the growth of Bifidobacterium longum and B. bifidum [7]. In mice, it stimulates the growth of bacteria of the family Ruminococcaceae and the genus Lactobacillus, at the expense of reducing pathogenic bacteria of the genera Peptococcus and Akkermansia [14]. The production of SCFAs in pigs implies benefits, such as protection against allergies [4,11,15].

We have evaluated the potential prebiotic effect of the fucoidan compound from the seaweed Fucus vesiculosus on the Lactobacillus reuteri growth to act against allergic symptoms.

\section{Results}

Absorbance measurements and bacterial counts allow for the corresponding growth curve of the bacteria under study to be obtained without the presence of fucoidan (control sample) over $48 \mathrm{~h}$ (see Figure 1). The latency phase of L. reuteri extends to $8 \mathrm{~h}$ of incubation. The exponential growth phase is observed from 8 to $24 \mathrm{~h}$ of incubation. The analysis of the bacterial growth when the Man Rogose Sharp [16] Broth (MRSB) medium is supplemented with fucoidan at two concentrations $(100$ and $2000 \mu \mathrm{g} / \mathrm{mL})$ provides interesting results. Figure 2 reflects the results of the growth evolution $(\log 10(\mathrm{~N} f)$, where $\mathrm{N} f=$ is the number of living microorganisms at each studied time (in Colony-Forming Units per milliliter $(\mathrm{CFU} / \mathrm{mL})$ ) both in the control sample and in that supplemented with fucoidan, at different concentrations. Fucoidan has a prebiotic effect on $L$. reuteri. The prebiotic effect is at its maximum, reaching $2.21 \pm 0.19$ cicles $\log 10$, at $48 \mathrm{~h}$ of incubation.

A multifactorial analysis of variance is performed to check whether the concentration and the exposure times had a significant effect on the prebiotic nature of the fucoidan. The conclusion was that both the exposure time $(p$-value $<0.01)$ and the fucoidan concentration ( $p$-value $<0.05)$ are factors with a significant effect on the prebiotic nature of the fucoidan from F. vesiculosus. In the case of exposure time to fucoidan as a factor of influence over $\log _{10}(\mathrm{~N} f)$, significant differences are observed between all measurements tested in the in vitro study, except exposure times between 24 and $26 \mathrm{~h}$. This result allows us to state that the prebiotic effect occurs after $24 \mathrm{~h}$ of incubation and increases moderately at $28 \mathrm{~h}$. In the case of the concentration of fucoidan as a factor of influence, significant differences are observed between the two concentrations, and also with respect to the control sample. After $48 \mathrm{~h}$ of exposure, even the fucoidan added at a relatively low concentration, $100 \mu \mathrm{g} / \mathrm{mL}$, produces an increase over the control sample, of about $0.9 \pm 0.6$ cicles $\log _{10}$. In the case of $2000 \mu \mathrm{g} / \mathrm{mL}$, the increase is about $2.21 \pm 0.19$ cicles $\log _{10}$. It can be concluded 
that fucoidan shows a clearer prebiotic effect the higher its concentration is, in the range (100-2000) $\mu \mathrm{g} / \mathrm{mL}$.

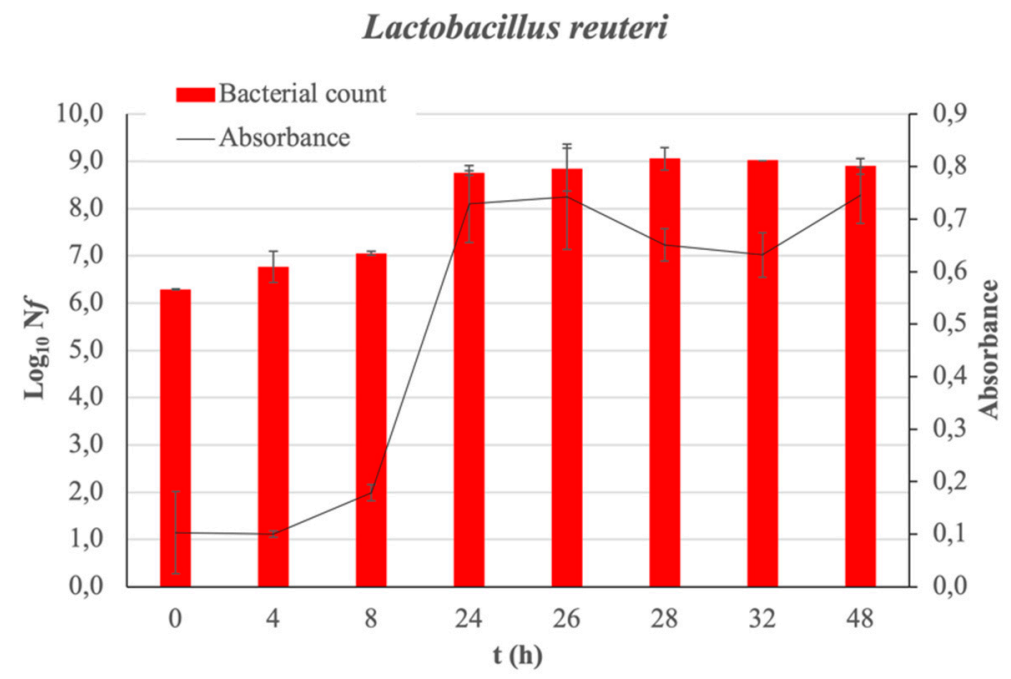

Figure 1. L. reuteri growth curve. The bar charts show the growth of bacteria in $\log _{10}(\mathrm{~N} f)$ as function of incubation time. Absorbance measured as a function of incubation time is also plotted with a line graph. In all cases, the standard deviation is represented.

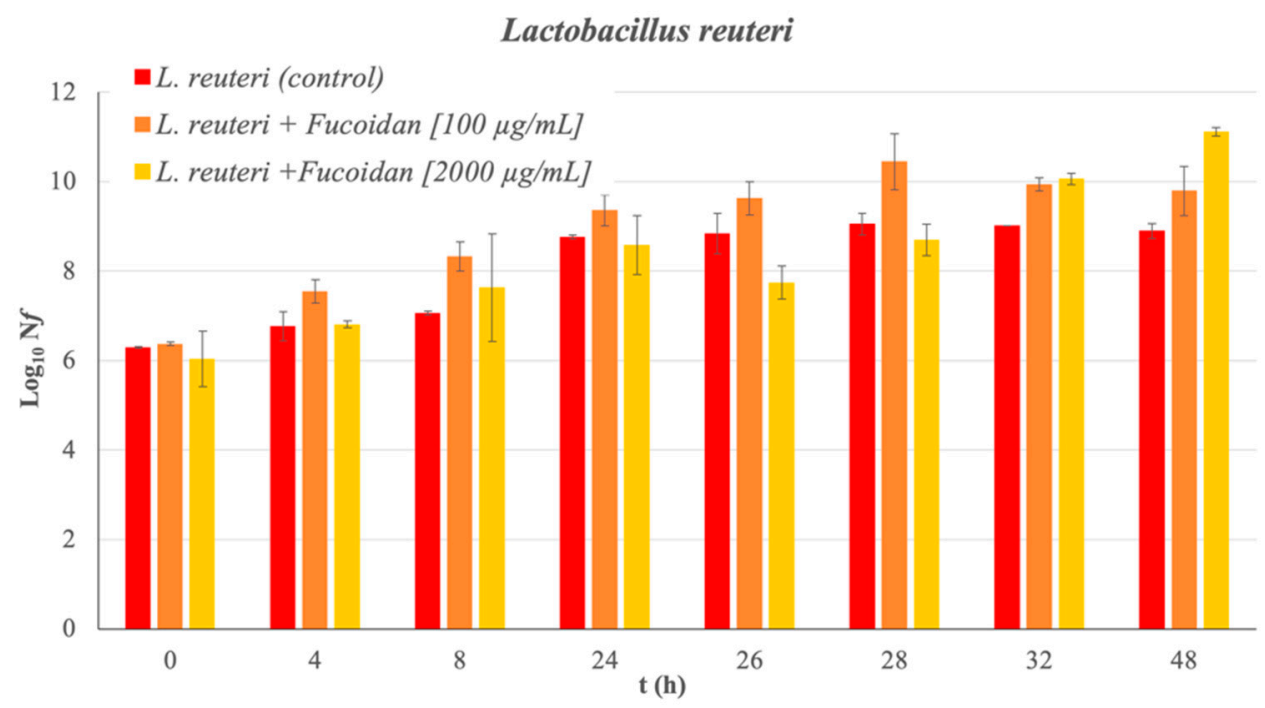

Figure 2. L. reuteri growth curve in different concentration of fucoidan. The bar charts show the growth of bacteria in $\log _{10}(\mathrm{~N} f)$ as function of incubation time.

These two factors (exposure time and concentration) are crucial to defining strategies for future usage of the compound in the formulation of prebiotic food and pharmaceutical products. The obtained in vitro results are promising, and they suggest that fucoidan has a clear prebiotic effect on beneficial bacteria for the health, and therefore could help to improve allergic symptoms.

\section{Discussion}

The action mode of probiotic bacteria is multifactorial: production of direct inhibitory components on pathogenic bacteria, decrease at luminal $\mathrm{pH}$ through the production of SCFAs, competition for nutrients with pathogenic bacteria or modulation of the immune response [17]. Rosenfeldt et al. [18] demonstrated that a combination of L. reuteri and $L$. 
rhamnosus, administered as a probiotic, could help by improving atopic dermatitis in children and by decreasing the appearance of eczema. Published studies on the prebiotic nature of fucoidan from F. vesiculosus seaweed are limited. However, Wang et al. [19] observed an increase in Lactobacillus spp. and Bifidobacterium spp. in rats after the ingestion of OS from brown seaweeds, over 14 days, at a concentration of $1 \%$ (weight/volume). Studies published by Palacios-Gorba et al. [20] demonstrated the antimicrobial nature of the fucoidan from F. vesiculosus, Undaria pinnatifida and Macrocystis pyrifera, against one of the most worrying pathogens at present, the Helicobacter pylori, showing both bacteriostatic and bactericidal effects. Tanino et al. [21] determined that fucoidan increases galectin-9 levels. This increase is responsible for its antiallergic action. Niki et al. [22] determined that galectin-9, secreted in blood by the fucoidan action, has the ability to attach to IgE, preventing the formation of the IgE-antigen complex by inducing an antiallergic effect. Fucoidan from F. vesiculosus is a "generally recognised as safe" ingredient and is beginning to be included in various products, such as dietary supplements to boost the immune system and as antioxidants.

\section{Materials and Methods}

\subsection{Culture Media and other Reagents}

The lactic acid bacteria were cultured in the liquid medium Man Rogose Sharp Broth (MRSB). The solid medium (MRSA) was prepared by adding $2 \%$ agar. Dilutions were performed in sterile peptone water ( $1 \% \mathrm{w} / \mathrm{V})$. Glycerol $(20 \%)$ was used to prepare the frozen cultures as a cryoprotective agent. The culture media were supplied by the Conda-Pronadisa Laboratories (Madrid, Spain).

\subsection{Bacterial Strains}

L. reuteri (CECT 925) was acquired from the Spanish Type Culture Collection (CECT) (Paterna, Spain). The culture was reactivated following the provider's instructions and inoculated in a $20 \mathrm{~mL}$ flask of sterile MRSB. It was stirred for $3 \mathrm{~h}$ at $37^{\circ} \mathrm{C}$. Afterwards, 480

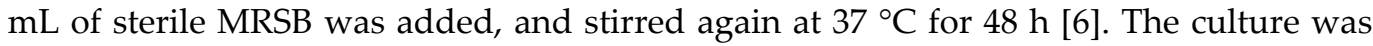
then centrifuged $(3000 \times \mathrm{g}), 10 \mathrm{~min}, 25^{\circ} \mathrm{C}$. After removing the supernatant, the pellet was resuspended in sterile MRSB and subjected to a new centrifugation cycle. The pellet from this centrifugation was reserved and resuspended in $50 \mathrm{~mL}$ of a solution of MRSB and glycerol [80:20] $(v / v)$. It was distributed in $2 \mathrm{~mL}$ cryovials, and these were stored frozen $\left(-80^{\circ} \mathrm{C}\right)$.

To determine the concentration of $L$. reuteri in the stored vials, three of them were taken and serial decimal dilutions were made in sterile peptone water $(1 \% w / v)$. The seeding of the different dilutions was performed in triplicate on MRSA plates. The mean result obtained from the count was $(3.0 \pm 0.6) \times 10^{9} \mathrm{CFU} / \mathrm{mL}$.

\subsection{Fucoidans}

Fucoidan lyophilisate from F. vesiculosus (95\% purity) was purchased from (SigmaAldrich). The preparation of the stock solutions consisted of dissolving $500 \mathrm{mg}$ of fucoidan powder in $50 \mathrm{~mL}$ of MRSB (concentration of $10 \mathrm{mg} / \mathrm{mL}$ ). They were distributed in $10 \mathrm{~mL}$ aliquots in five sterile tubes and kept frozen $\left(-80^{\circ} \mathrm{C}\right)$ until the time of use.

\subsection{Turbidimetry, Growth Curves and Plate Count}

Measurements were performed on a spectrophotometer (T60U Spectrometer, PG Instruments Ltd.) at $\lambda=595 \mathrm{~nm}$. The measured absorbance is correlated with the concentration of bacteria $\left(\log _{10}()\right)$. Precultures were prepared by diluting $100 \mu \mathrm{L}$ of bacterial glycerin in $9.9 \mathrm{~mL}$ of MRSB. The solution was left to incubate at $28^{\circ} \mathrm{C}$ for $72 \mathrm{~h}$.

Control sample preparation consisted of mixing $20 \mu \mathrm{L}$ of pre-culture and $19.98 \mathrm{~mL}$ of sterile MRSB. The fucoidan trial at $100 \mu \mathrm{g} / \mathrm{mL}$ was prepared by mixing $20 \mu \mathrm{L}$ of preculture, $200 \mu \mathrm{L}$ of fucoidan stock solution and $19.78 \mathrm{~mL}$ of sterile MRSB. The fucoidan trial 
at $2000 \mu \mathrm{g} / \mathrm{mL}$ was prepared by mixing $20 \mu \mathrm{L}$ of the pre-culture, $4 \times 103 \mu \mathrm{L}$ of fucoidan stock solution and $15.98 \mathrm{~mL}$ of sterile MRSB. All cultures were incubated during $48 \mathrm{~h}$ in an oven at $28{ }^{\circ} \mathrm{C}$. A sampling was performed periodically to monitor and record the growth of the bacteria, both in the control sample and in fucoidan trials. Afterwards, $1 \mathrm{~mL}$ aliquots from the culture were taken in triplicate and the corresponding absorbances were measured. To obtain the bacterial concentration as a function of time, serial decimal dilutions and MRSA seeding were performed at different times during the incubation period. They were incubated in an oven at $28{ }^{\circ} \mathrm{C}$ for $48 \mathrm{~h}$. Graphs were built to correlate concentration with time and absorbance.

\subsection{Mathematical and Statistical Analysis}

The growth curves and bar charts were built with the Excel program (Microsoft, Redmond, Washington, USA). An ANOVA (multifactorial analysis of variance) analysis was performed with the Statgraphics 18 Centurion program (Statgraphics Technologies Inc., The Plains, Virginia, USA), in order to detect significant differences $(p$-value $<0.05)$ between bacterial growth $\left(\log _{10}(\mathrm{~N} f), \log _{10}(\mathrm{CFU} / \mathrm{mL})\right)$ under the effect of the different factors studied (fucoidan concentration, exposure time, Lactobacillus strain).

\section{Conclusions}

Fucoidan from Phaeophyceae seaweed shows a prebiotic capacity on beneficial microorganisms, in improving allergic symptoms, and also shows antimicrobial capacity against pathogens in the gastrointestinal tract. Fucoidan from F. vesiculosus shows, in vitro, a maximum prebiotic potential equivalent to $2.21 \pm 0.19 \log _{10}$ cycles on L. reuteri (applied at a concentration of $2000 \mu \mathrm{g} / \mathrm{mL}$ ). The combination of the prebiotic effect of fucoidan and its direct action on allergies and other health problems make this molecule a perfect nutraceutical candidate, with a very promising future in both the pharmaceutical and food industries.

\section{Institutional Review Board Statement: Not applicable.}

Informed Consent Statement: Not applicable.

Data Availability Statement: Data available on request.

Acknowledgments: The present research work has been supported by funds provided by the Generalitat Valenciana (GVA), Conselleria de Innovación, Universidades, Ciencia y Sociedad Digital with reference GV2020/031 under the project grants supporting emergent research groups in R\&D\&I.

Conflicts of Interest: The authors declare no conflict of interest.
Abbreviations
GM Gut microbiota
PS Polysacchacarides
OS Oligosaccharides
SCFAsShort chain fatty acids
MRSB Man Rogose Sharp Broth
MRSAMan Rogose Sharp agar
CFU Colony forming units

The following abbreviations are used in this manuscript:

\section{References}

1. Belkaid, Y.; Harrison, O.J. Homeostatic Immunity and the Microbiota. Immunity 2017, 46, 562-576, doi:10.1016/j.immuni.2017.04.008.

2. Tinahones, F.J. La importancia de la microbiota en la obesidad. Rev. Esp. Endocrinol. Pediatr. 2017, 8, 16-20.

3. Icaza-Chávez, M. Microbiota intestinal en la salud y la enfermedad. Rev. Gastroenterol. México 2013, 78, 240-248, doi:10.1016/j.rgmx.2013.04.004. 
4. Lee, K.H.; Song, Y.; Wu, W.; Yu, K.; Zhang, G. The gut microbiota, environmental factors, and links to the development of food allergy. Clin. Mol. Allergy 2020, 18, 5, doi:10.1186/s12948-020-00120-x.

5. Zhuang, L.; Chen, H.; Zhang, S.; Zhuang, J.; Li, Q.; Feng, Z. Intestinal Microbiota in Early Life and Its Implications on Childhood Health. Genom. Proteom. Bioinform. 2019, 17, 13-25, doi:10.1016/j.gpb.2018.10.002.

6. Li, L.; Fang, Z.; Liu, X.; Hu, W.; Lu, W.; Lee, Y.k.; Zhao, J.; Zhang, H.; Chen, W. Lactobacillus reuteri attenuated allergic inflammation induced by HDM in the mouse and modulated gut microbes. PLoS ONE 2020, 15, e0231865, doi:10.1371/journal.pone.0231865.

7. Zaporozhets, T.S.; Besednova, N.N.; Kuznetsova, T.A.; Zvyagintseva, T.N.; Makarenkova, I.D.; Kryzhanovsky, S.P.; Melnikov, V.G. The prebiotic potential of polysaccharides and extracts of seaweeds. Russ. J. Mar. Biol. 2014, 40, 1-9, doi:10.1134/S1063074014010106.

8. Hu, B.; Gong, Q.; Wang, Y.; Ma, Y.; Li, J.; Yu, W. Prebiotic effects of neoagaro-oligosaccharides prepared by enzymatic hydrolysis of agarose. Anaerobe 2006, 12, 260-266, doi:10.1016/j.anaerobe.2006.07.005.

9. Han, Z.L.; Yang, M.; Fu, X.D.; Chen, M.; Su, Q.; Zhao, Y.H.; Mou, H.J. Evaluation of Prebiotic Potential of Three Marine Algae Oligosaccharides from Enzymatic Hydrolysis. Mar. Drugs 2019, 17, 173, doi:10.3390/md17030173.

10. Li, B.; Lu, F.; Wei, X.; Zhao, R. Fucoidan: Structure and Bioactivity. Molecules 2008, 13, 1671-1695, doi:10.3390/molecules13081671.

11. de Jesus Raposo, M.F.; de Morais, A.M.M.B.; de Morais, R.M.S.C. Emergent Sources of Prebiotics: Seaweeds and Microalgae. Mar. Drugs 2016, 14, 27, doi:10.3390/md14020027.

12. Luthuli, S.; Wu, S.; Cheng, Y.; Zheng, X.; Wu, M.; Tong, H. Therapeutic Effects of Fucoidan: A Review on Recent Studies. Mar. Drugs 2019, 17, 487, doi:10.3390/md17090487.

13. Murphya, P.; Bello, F.D.; O’Doherty, J.; Arendt, E.K.; Sweeney, T.; Coffey, A. The effects of liquid versus spray-dried Laminaria digitata extract on selected bacterial groups in the piglet gastrointestinal tract (GIT) microbiota. Anaerobe 2013, 21, 1-8, doi:10.1016/j.anaerobe.2013.03.002.

14. Shang, Q.; Shan, X.; Cai, C.; Hao, J.; Li, G.; Yu, G. Dietary fucoidan modulates the gut microbiota in mice by increasing the abundance of Lactobacillus and Ruminococcaceae. Food Funct. 2016, 7, 3224-3232, doi:10.1039/C6FO00309E.

15. O'Doherty, J.; Dillon, S.; Figat, S.; Sweeney, T. The effects of lactose inclusion and seaweed extract derived from Laminaria spp. on performance, digestibility of diet components and microbial populations in newly weaned pigs. Anim. Feed Sci. Technol. 2010, 157, 173-180, doi:10.1016/j.anifeedsci.2010.03.004.

16. De Man, J.C.; Rogosa, M.; Sharpe, M.E. A medium for the cultivation of Lactobacilli. J. Appl. Bacteriol. 1960, 23, 130-135, doi:10.1111/j.1365-2672.1960.tb00188.x.

17. Tuohy, K.; Probert, H.; Smejkal, C.; Gibson, G. Using probiotics and prebiotics to improve gut health. Drug Discov. Today 2003, 8, 692-700, doi:10.1016/S1359-6446(03)02746-6.

18. Rosenfeldt, V.; Benfeldt, E.; Nielsen, S.D.; Michaelsen, K.F.; Jeppesen, D.L.; Valerius, N.H.; Paerregaard, A. Effect of probiotic Lactobacillus strains in children with atopic dermatitis. J. Allergy Clin. Immunol. 2003, 111, 389-395, doi:10.1067/mai.2003.389.

19. Wang, Y.; Han, F.; Hu, B.; Li, J.; Yu, W. In vivo prebiotic properties of alginate oligosaccharides prepared through enzymatic hydrolysis of alginate. Nutr. Res. 2006, 26, 597-603, doi:10.1016/j.nutres.2006.09.015.

20. Palacios-Gorba, C.; Pina, R.; Tortajada, M.; Jiménez-Belenguer, A.; Siguemoto, E.; Rodrigo, D.; Pina-Pérez, M. Caenorhabditis elegans as in vivo model to assess the FUCOIDAN bioactivity preventing Helicobacter pylori infection. Food Funct. 2020, 11, 45254534, doi:10.1039/D0FO00768D.

21. Tanino, Y.; Hashimoto, T.; Ojima, T.; Mizuno, M. F-fucoidan from Saccharina japonica is a novel inducer of galectin-9 and exhibits anti-allergic activity. J. Clin. Biochem. Nutr. 2016, 59, 25-30, doi:10.3164/jcbn.15-144.

22. Niki, T.; Tsutsui, S.; Hirose, S.; Aradono, S.; Sugimoto, Y.; Takeshita, K.; Nishi, N.; Hirashima, M. Galectin-9 Is a High Affinity IgE-binding Lectin with Anti-allergic Effect by Blocking IgE-Antigen Complex Formation. J. Biol. Chem. 2009, 284, 32344-32352, doi:10.1074/jbc.M109.035196. 